\title{
Baryon Number Violating Transitions in String Backgrounds
}

\author{
Warren B. Perkins \\ Department of Physics \\ University of Wales, Swansea \\ Singleton Park \\ Swansea, SA2 8PP
}

\begin{abstract}
We construct field configurations that interpolate between string background states of differing baryon number. Using these configurations we estimate the effect of the background fields on the energy barrier separating different vacua. In the background of a superconducting GUT string the energy barrier is increased, while in an electroweak string background or the electroweak layer of a non-superconducting string the energy barrier is reduced. The energy barrier depends sensitively on both the background gauge and scalar fields.
\end{abstract}




\section{1) Intoduction}

The realisation that electroweak baryon number violation can occur at an appreciable rate in the early universe has raised the hope of explaining the observed baryon asymmetry of the universe with $\mathrm{TeV}$ scale physics. Most models rely on a first order phase transition that proceeds via the nucleation and subsequent growth of true vacuum bubbles[1]. The order of this phase transition is not certain and it is not clear that it is sufficiently strongly first order for these mechanisms to work[2]. This provides an incentive to produce scenarios that do not rely on the order of the electroweak transition. Just such a method has been suggested by Davis, Brandenberger and Trodden[3] who make use of the decay of a preexisting string network to provide the departure from thermal equilibrium. Just as in most methods based on bubble nucleation, an extension of the standard model is required to increase CP violation, this is provided by extending the Higgs sector of the theory. The coupling of the extra Higgs field is such as to generate a chemical potential in regions that are entering or leaving the core of the string. This chemical potential leads to the generation of antibaryons at the leading edge of the string and baryons on its trailing edge. The decay of the string network causes a greater volume of space to leave the string core region than enter it, leading to a net production of baryons. As the antibaryons are produced on the leading edge of the string, they enter the core region shortly after production and baryon production is enhanced if they can decay before the string leaves them behind. If the baryon asymmetry is to survive, the rate of baryon number violation outside the string should be small, that is we are in an epoch when the sphaleron energy is large compared to the temperature so that the sphaleron transition rate [4]

$$
\Gamma_{\Delta B}=\gamma\left(\alpha_{w k} T\right)^{-3} M_{W}^{7} \mathrm{e}^{\left(-4 \pi \nu(T) / g_{w k} T\right)}
$$

is Boltzmann suppressed away from the string core. Here $\alpha_{w k}$ is the $\mathrm{SU}(2)$ fine structure constant, $M_{W}$ the mass of the $\mathrm{W}$ and $\nu(T)$ the expectation value of the Higgs field. For this mechanism to work baryon number violating processes must occur in the core of the string at a greater rate than outside the string.

The first step to finding the rate of baryon number violating transitions is to calculate the height of the energy barrier that separates the background states with different baryon number. If the barrier height is increased relative to the homogeneous background case, the rate of baryon number violation will probably be even more suppressed. On the other hand, if the barrier height is decreased, the exponential term in the rate will be decreased, but the prefactors must be calculated to obtain a firm result for the rate.

In this paper we construct field space paths connecting purely bosonic string background states with different baryon numbers. The energy barrier obtained from each path provides an upper bound to the barrier that would be obtained from the minimum action path.

In section 2 we review the construction on the ordinary sphaleron in a form that can be applied to string background fields. This method is then applied to various string backgrounds: superconducting string background in section 3 and electroweak string background in section 4. An alternative for the superconducting string background is discussed 
in section 5 and we conclude in section 6 . The calculations of baryon number change and energy barriers are presented in appendices A and B respectively. 


\section{2) The Ordinary Sphaleron}

In this section we review the construction and properties of the electroweak sphaleron [5]. The construction is presented in a form that is readily generalised to string backgrounds and the various contributions to the sphaleron energy are highlighted.

We take the bosonic part of the electroweak lagrangian to be

$$
\mathcal{L}=-\frac{1}{4} Y_{\mu \nu} Y^{\mu \nu}-\frac{1}{4} W_{\mu \nu}^{a} W^{a \mu \nu}+\left(D_{\mu} \Phi\right)^{\dagger} D^{\mu} \Phi-\lambda\left(\Phi^{\dagger} \Phi-\frac{\nu^{2}}{2}\right)^{2}
$$

where $\mathrm{W}$ is the $\mathrm{SU}(2)$ triplet of gauge fields and $\mathrm{Y}$ is the $\mathrm{U}(1)$ gauge field. Using $\mathrm{g}$ and $\mathrm{g}$ ' to denote the $\mathrm{SU}(2)$ and $\mathrm{U}(1)$ coupling constants, the $\mathrm{U}(1)$ field strength is given by

$$
Y_{\mu \nu}=\partial_{\mu} Y_{\nu}-\partial_{\nu} Y_{\mu}
$$

the $\mathrm{SU}(2)$ field strength by

$$
W_{\mu \nu}^{a}=\partial_{\mu} W_{\nu}^{a}-\partial_{\nu} W_{\mu}^{a}-g \epsilon^{a b c} W_{\mu}^{b} W_{\nu}^{c},
$$

and the covariant derivative for the Higgs doublet $\Phi$ by

$$
D_{\mu}=\partial_{\mu}+\frac{i}{2} g \sigma^{a} W_{\mu}^{a}+\frac{i}{2} g^{\prime} Y_{\mu}
$$

where $\sigma^{a}$ are the Pauli matrices.

In the case of vanishing Weinberg angle the procedure for constructing a nontrivial path from the ordinary vacuum, through the sphaleron back to the ordinary vacuum is based on a unitary matrix, U. Using the notation of ref.6 we obtain the nontrivial path from the trivial vacuum configuration: $\Phi^{\dagger}=(0, v / \sqrt{2}), W=Z=A=0$, by first considering the transformation

$$
\Phi \rightarrow \tilde{\Phi}=U \Phi \quad W_{\mu} \rightarrow-\frac{1}{g}\left(\partial_{\mu} U\right) U^{-1}
$$

where

$$
U=\left(\begin{array}{cc}
\mathrm{e}^{i \mu}(\cos \mu-i \sin \mu \cos \theta) & \sin \mu \sin \theta \mathrm{e}^{i \phi} \\
-\sin \mu \sin \theta \mathrm{e}^{-i \phi} & \mathrm{e}^{-i \mu}(\cos \mu+i \sin \mu \cos \theta)
\end{array}\right),
$$

$\theta$ and $\phi$ are the usual spherical polar angles and $\mu$ is a parameter. $\mathrm{U}$ is an element of $\mathrm{SU}(2)$ and so this transformation would constitute a gauge transformation if it could be applied everywhere. However, if we simply apply the transformation everywhere, the Higgs field is not single valued on the polar axis and the gauge fields are divergent. To remove these problems profile functions are introduced:

$$
\begin{gathered}
\Phi=(1-h(r))\left(\begin{array}{c}
0 \\
\frac{v}{\sqrt{2}} \mathrm{e}^{-i \mu} \cos \mu
\end{array}\right)+h(r) \tilde{\Phi} \\
W_{\mu}=-\frac{f(r)}{g}\left(\partial_{\mu}\right) U U^{-1}
\end{gathered}
$$


The boundary conditions on $h$ and $f$ are, $h, f \rightarrow 0$ as $r \rightarrow 0$ and $h, f \rightarrow 1$ as $r \rightarrow \infty$. The resulting field configuration is single valued and finite. As the transformation differs from a gauge transformation only when the profile functions differ from unity, the energy density of the configuration is localised and the total energy is finite.

The asymptotic Higgs field is given by $\tilde{\Phi}$,

$$
\tilde{\Phi}=\frac{v}{\sqrt{2}}\left(\begin{array}{c}
\sin \mu \sin \theta \mathrm{e}^{i \phi} \\
e^{-i \mu}(\cos \mu+i \sin \mu \cos \theta)
\end{array}\right)
$$

The nontrivial nature of this transformation is apparent if we consider the winding of the asymptotic Higgs field around the vacuum manifold. We are interested in the Higgs field on some large shell surrounding the sphaleron. Firstly the asymptotic Higgs field remains on the vacuum manifold,

$$
\lim _{r \rightarrow \infty} \tilde{\Phi}^{\dagger} \tilde{\Phi}=v^{2} / 2
$$

and in general we can find one set of values for $\mu, \theta$ and $\phi$ that correspond to each point on the vacuum manifold. Thus the asymptotic Higgs field covers the vacuum manifold once during the transition from $\mu=0$ to $\mu=\pi$.

The change in baryon plus lepton number during the transition can also be calculated using the anomaly equation for general Weinberg angle,

$$
\partial_{\mu} J_{B+L}^{\mu}=\frac{N_{f}}{16 \pi^{2}}\left(g^{2} W_{a}^{\mu \nu} \tilde{W}_{a \mu \nu}-g^{\prime 2} Y^{\mu \nu} \tilde{Y}_{\mu \nu}\right)
$$

where $N_{f}$ is the number of families.

Integrating over all space and the time interval of the transition and assuming that we can drop the boundary terms, we have

$$
\Delta(B+L)=\int d^{4} x\left(\frac{N_{f}}{16 \pi^{2}}\left(g^{2} W_{a}^{\mu \nu} \tilde{W}_{a \mu \nu}-g^{\prime 2} Y^{\mu \nu} \tilde{Y}_{\mu \nu}\right)\right)
$$

where $N_{f}$ is the number of families. Explicit calculation with the field configurations given above yields (see appendix A)

$$
\Delta(B+L)=2 N_{f}
$$

Thus the transition not only covers the vacuum manifold but also produces $N_{f}$ baryons.

This process is greatly suppressed at low temperatures due to the energy barrier separating the two vacua. The saddle point configuration between the two vacua is called the sphaleron and is the field configuration at $\mu=\pi / 2$. The energy density of the sphaleron configuration is (see appendix B)

$$
\begin{aligned}
\mathcal{E}_{\text {sphaleron }}= & \frac{\nu^{2}}{2} \frac{\partial h^{2}}{\partial r}+\frac{\nu^{2}}{r^{2}} h^{2}(1-f)^{2} \\
& +4\left(\frac{\partial f}{\partial r}\right)^{2} \frac{1}{g^{2} r^{2}}+\frac{8}{r^{4} g^{2}}(1-f)^{2} f^{2}+\lambda \frac{\nu^{4}}{4}\left(1-h^{2}\right)^{2}
\end{aligned}
$$


This leads to a sphaleron energy of the form[5]

$$
E_{\text {Sphaleron }}=\frac{8 \pi M_{W}}{g^{2}} D\left(\frac{\lambda}{g^{2}}\right)
$$

where $\mathrm{D}$ is a dimensionless factor. Numerical integration yields $[5] \mathrm{D}(0)=1.52, \mathrm{D}(1)=2.07$ and $\mathrm{D}(\infty)=2.70$.

The large barrier between the vacua of different winding numbers leads to a vast suppression of baryon number violating processes at low temperatures. At high temperatures the Boltzmann suppression is reduced and at very high temperatures the rate becomes $\mathrm{O}(\alpha T)^{4}[7]$.

Strictly speaking the sphaleron is the field configuration that corresponds to the saddle point separating the two distinct vacua. The sphaleron thus has the lowest possible energy of any configuration that has the maximum energy on a given path. In the general setting we will refer to the highest energy configuration on a path as the sphaleron although we do not show that this is in fact the saddle point. In other words we will construct upper bounds on the energy barrier separating the background states. find

If we scale the radial variable in our expression for the sphaleron energy, $r \rightarrow \eta x$, we

$$
\begin{aligned}
E_{\text {sphaleron }}=4 \pi \eta \int d x & {\left[\frac{\nu^{2}}{2}\left(x^{2} \frac{\partial h^{2}}{\partial x}+2 h^{2}(1-f)^{2}\right)\right.} \\
& +4\left(\frac{\partial f}{\partial x}\right)^{2} \frac{1}{\eta^{2} g^{2}}+\frac{8}{\eta^{2} x^{2} g^{2}}(1-f)^{2} f^{2} \\
& \left.+\lambda \frac{\nu^{4}}{4} \eta^{2} x^{2}\left(1-h^{2}\right)^{2}\right]
\end{aligned}
$$

Extremising the energy with respect to the arbitrary scale factor allows us to express the pure gauge field contributions to the energy in terms of those involving the Higgs field:

$$
\int d x\left[\frac{\nu^{2}}{2}\left(x^{2} \frac{\partial h^{2}}{\partial x}+2 h^{2}(1-f)^{2}\right)+3 \lambda \frac{\nu^{4}}{4} \eta^{2} x^{2}\left(1-h^{2}\right)^{2}\right]=\int d x\left[4\left(\frac{\partial f}{\partial x}\right)^{2} \frac{1}{\eta^{2} g^{2}}+\frac{8}{\eta^{2} x^{2} g^{2}}(1-f)^{2} f^{2}\right]
$$

The sphaleron energy has two components, one coming purely from the gauge fields and the other from the Higgs sector. As in the case of gauge topological defects, we can think of the scalar field configuration being supported against collapse by the gauge field energy trapped in the core of the object. With this picture in mind we see why sphaleron transitions in string backgrounds might not be as energetically disfavoured as those in a trivial background. In the core of a string the electroweak symmetry is restored, the electroweak Higgs field is already forced to zero at the core of the defect, so at least this component of the sphaleron energy has already been 'paid for' by the string. If there is a region of electroweak symmetry restoration that is sufficiently large to accommodate the Higgs profile of the sphaleron we might expect a reduction in the energy barrier by a factor of about 0.5 . If the region of symmetry restoration is much larger, as is the case for 
superconducting cosmic strings, we might expect further reductions in the energy barrier as the gauge fields can become more diffuse.

However, we must always bear in mind the cause of the symmetry restoration, in the case of superconducting strings the gauge field generated by the current not only gives a large region of symmetry restoration but also couples to the sphaleron fields. This situation is discussed in the next section. 


\section{3) Baryon Number Violating Transitions In A Superconducting String Back- ground}

In the next two sections we construct field space paths that connect string states with different baryon numbers. The paths are constructed using the method outlined for the vacuum to vacuum transitions. In each case we consider the change in baryon number and, most importantly, the energy barrier separating the initial and final states. We consider three cases, the superconducting string, the electroweak string and the electroweak layer of a nonsuperconducting string formed at some transition above the electroweak scale. In each case we apply the transformation matrix $U$ discussed above and introduce profile functions to ensure that the fields are finite and single valued everywhere.

In this section we consider the superconducting string background. The possibility of an enhanced sphaleron transition rate in the region of electroweak symmetry restoration around a superconducting string was suggested by Damgaard and Esprin[8]. In contrast to the background fields discussed below, the background gauge field considered in ref. 8 was pure hypercharge.

Our first task is to construct the background state that will form the endpoints of our path. In this section we consider the background electroweak fields provided by a superconducting GUT string. A model of the electroweak fields around a GUT superconducting string is discussed in detail in ref.9, here we briefly review the main features of the field configurations. In this model the string carries a hypercharge current and so provides a source for both the electromagnetic and the $\mathrm{Z}$ fields. The electroweak fields around the string have the form:

$$
W_{\mu}^{ \pm}=0 \quad \Phi=\left(\begin{array}{c}
0 \\
\phi(\rho)
\end{array}\right) \quad Z_{\mu}=\delta_{\mu, z} Z(\rho) \quad A_{\mu}=\delta_{\mu, z} A(\rho)
$$

where $\rho$ is the radial coordinate in a cylindrical polar coordinate system. The electromagnetic gauge field is free everywhere outside the string and so has a logarithmic form out to scales where the string curvature becomes important. The large $\mathrm{Z}$ field produced by the hypercharge current provides a large, positive contribution to the Higgs field mass which leads to symmetry restoration in some region around the string. Inside the symmetry restored region the $\mathrm{Z}$ field is massless and behaves logarithmically, outside the region of symmetry restoration the $\mathrm{Z}$ field becomes massive and decays exponentially. For large string currents the profiles of the Higgs and $\mathrm{Z}$ fields can be modelled as

$$
\begin{gathered}
\phi=\frac{1}{2}\left(1+\tanh k\left(\rho-\rho_{0}\right)\right) \frac{\nu}{\sqrt{2}} \\
Z_{3}= \begin{cases}\frac{I}{2 \pi} \log \left(\frac{\rho}{\beta}\right) & \rho<\rho_{0} \\
a K_{0}\left(\frac{1}{2} G \nu \rho\right) & \rho>\rho_{0}\end{cases}
\end{gathered}
$$

and the three parameters $r_{0}, \mathrm{k}$ and $\beta$ set to minimise the energy of the configuration. For large currents this yields,

$$
\rho_{0}=\frac{I g}{2 \pi M_{H} M_{W}}, \quad \beta \simeq \rho_{0} \mathrm{e}^{2 / \nu G \rho_{0}} \simeq \rho_{0}
$$


The important features of these field configurations are the large region of symmetry restoration and the large background gauge field. For a maximal string current, $I \sim 10^{20}$ amps, the region of symmetry restoration has a radius $r \sim 10^{10} \mathrm{GeV}^{-1} \sim 10^{-5} \mathrm{~m}$. The size of the symmetry restored region has been confirmed numerically [10]. The form of the $W^{3}$ and hypercharge fields are important for the energetics of the baryon number violating path. To find these we must also model the photon field around the string. As the photon field is free everywhere outside the GUT string, we take its profile to be

$$
A_{z}(\rho)=\frac{\tilde{I}}{2 \pi} \log \left(\frac{\rho}{\gamma}\right)
$$

We can find $\tilde{I}$ in terms of $I$ using the relationships between the $\mathrm{Z}$ and $\mathrm{A}$ fields and the original $\mathrm{SU}(2)$ and hypercharge fields.

$$
\left(\begin{array}{l}
Z \\
A
\end{array}\right)=\left(\begin{array}{cc}
-\cos \theta_{W} & \sin \theta_{W} \\
\sin \theta_{W} & \cos \theta_{W}
\end{array}\right)\left(\begin{array}{c}
W^{3} \\
Y
\end{array}\right)
$$

Thus in the symmetry restored region we have

$$
G W^{3}=\frac{-I g}{2 \pi} \log \left(\frac{\rho}{\beta}\right)+\frac{\tilde{I} g^{\prime}}{2 \pi} \log \left(\frac{\rho}{\gamma}\right) .
$$

The string does not act as source for $W^{3}$ and the field is free in the region of symmetry restoration, thus $W^{3}$ should be constant in this region. This leads to

$$
A_{z}(\rho)=\frac{g I}{2 \pi g^{\prime}} \log \left(\frac{\rho}{\gamma}\right) .
$$

The scale in the logarithm cannot be determined for an infinitely long, straight string. However, if we imagine the string to be curved we can determine a value for $\gamma$. In particular, if we consider the string to form a circular loop of radius $R$, the natural value for $\gamma$ is of order $R[11]$. As the typical curvature scales are of order kiloparsecs, this curvature has no further appreciable affect. The forms for the SU(2) and hypercharge fields are then,

$$
\begin{aligned}
& Y_{z}= \begin{cases}\frac{I}{2 \pi} \frac{1}{G g^{\prime}}\left(g^{\prime 2} \log \left(\frac{\rho}{\beta}\right)+g^{2} \log \left(\frac{\rho}{\gamma}\right)\right) & \rho<\rho_{0} \\
\frac{I}{2 \pi} \frac{g^{2}}{G g^{\prime}} \log \left(\frac{\rho}{\gamma}\right)+\frac{g^{\prime}}{G} a K_{0}\left(\frac{1}{2} G \nu \rho\right) & \rho>\rho_{0}\end{cases} \\
& W_{z}^{3}= \begin{cases}\frac{I}{2 \pi} \frac{g}{G} \log \left(\frac{\beta}{\gamma}\right) & \rho<\rho_{0} \\
\frac{I}{2 \pi} \frac{g}{G} \log \left(\frac{\rho}{\gamma}\right)-\frac{g}{G} a K_{0}\left(\frac{1}{2} G \nu \rho\right) & \rho>\rho_{0}\end{cases}
\end{aligned}
$$

Thus $W_{z}^{3}$ is large and constant within the symmetry restored region.

The most naive generalisation of the procedure outlined in section 2 for the production of baryon number violating paths is to apply the same transformation $\mathrm{U}$ as in the vacuum case. As we will see, this produces a transformation with the required change in baryon number, however we have no guarantee that this is the lowest energy path between the 
string background states. Indeed, given the different symmetries of the string and ordinary sphaleron it seems unlikely that this procedure will generate the lowest energy path, even for zero Weinberg angle. We will return to this point once we have calculated the energy of the sphaleron derived from this naive approach.

Applying the transformation discussed in section 2 to the asymptotic Higgs field we find,

$$
\Phi \rightarrow U \Phi=\frac{\nu}{\sqrt{2}}\left(\begin{array}{c}
\sin \mu \sin \theta \mathrm{e}^{i \phi} \\
\mathrm{e}^{-i \mu}(\cos \mu+i \sin \mu \cos \theta)
\end{array}\right) p
$$

where $p$ is the profile function for the Higgs field which satisfies the boundary conditions $p \rightarrow 0$ as $\rho \rightarrow 0$ and $p \rightarrow 1$ as $\rho \rightarrow \infty$. Apart from the region close to the string core the Higgs field lies on its vacuum manifold and we can show, as in the case of the ordinary sphaleron, that every point on the vacuum manifold is covered at some stage during the transition by the Higgs field at some point on any large shell around the sphaleron. Possible complications arise as the string will always pierce this large shell along the polar axis. The fact that the Higgs field vanishes at both $\theta=0$ and $\theta=\pi$ means that the lines on the vacuum manifold that were covered by the north and south poles are no longer covered. The north pole, $\cos \theta=1$, is invariant during the transition while the south pole, $\cos \theta=-1$ is mapped to

$$
\frac{\nu}{\sqrt{2}}\left(\begin{array}{c}
0 \\
\mathrm{e}^{-2 i \mu}
\end{array}\right)
$$

Apart from this line the whole vacuum manifold is covered.

We can verify that this transition violates baryon number by explicitly calculating $\Delta(B+L)$. The gauge fields in this case are given by

$$
\vec{W}_{\mu} \rightarrow U \tau^{a} W_{\mu}^{a} U^{-1}-\frac{f}{g}\left(\partial_{\mu} U\right) U^{-1}=U \delta_{\mu, z} \tau^{3} W_{z}^{3(\text { string })} U^{-1}-\frac{f}{g}\left(\partial_{\mu} U\right) U^{-1}
$$

where we have again introduced a profile function, $f . W_{z}^{3 \text { (string) }}$ is the gauge field generated by the current in the string, it satisfies the boundary conditions, $W_{z}^{3 \text { (string) }}(\rho=0)=$ const. and $W_{z}^{3 \text { (string) }}(\rho \rightarrow \infty) \rightarrow \frac{I}{2 \pi} \frac{g}{G} \log \left(\frac{\rho}{\gamma}\right)$. In this case the cylindrical symmetry of the string and the spherical nature of the transformation suggest that we let $f$ depend on both $\theta$ and $\mathrm{r}$ (we choose to work in spherical polars). Similarly for $\mu \neq 0$ we allow the Higgs field profile function $p$ to depend on both $\theta$ and $\mathrm{r}$. Generalising the profiles in this way is our only concession to the mixed nature of the symmetries of the problem.

We can explicitly calculate the change in baryon plus lepton number that the transformation induces by substituting the gauge field configurations into the anomaly equation (see App.A). We find

$\operatorname{Tr}\left(W_{t r} W_{\theta \phi}-W_{t \theta} W_{r \phi}+W_{t \phi} W_{r \theta}\right)=$ pure sphaleron contribution $+\sin (\mu) \cos (\mu)(\mu$ indep. $)$

where the pure sphaleron contribution is as discussed in the previous section;

$$
12(\cos (\theta)-1) \sin ^{2}(\mu) \frac{\partial f}{\partial r} \frac{f(1-f)}{r^{2} g^{2}}
$$


Integrating from $\mu=0$ to $\mu=\pi$ removes the contribution from the string gauge field, thus the string gauge field does not alter the change in baryon number caused by the transition. We now have a path that interpolates between string background states of different baryon number.

The energy density associated with the static field configurations at any point on this path is calculated in App.B. It is convenient to work with $W(r, \theta)=g W_{z}^{3}(r, \theta)$ and $Y(r, \theta)=g^{\prime} Y_{z}(r, \theta)$ when calculating the energy of the sphaleron. The boundary conditions on $W$ are then simply $W \rightarrow$ constant at the string core and $W \sim \log (r \sin \theta / \gamma)$ far from the string. The energy density is found to be:

$$
\begin{aligned}
\mathcal{E}= & \frac{\partial f^{2}}{\partial r} \frac{\sin ^{2} \mu}{g^{2} r^{2}}+\frac{8}{r^{4} g^{2}} \sin ^{4} \mu(1-f)^{2} f^{2}+\frac{2}{g^{2} r^{4}} \sin \mu^{2} \frac{\partial f^{2}}{\partial \theta} \\
& +\frac{1}{2 g^{2} r^{2}}\left(\sin \theta r \frac{\partial W}{\partial r}+\cos \theta \frac{\partial W}{\partial \theta}\right)^{2} \\
& +\frac{2}{g^{2} r^{2}} \frac{\partial f}{\partial r} \sin \mu \cos \mu \sin \theta\left(\sin \theta r \frac{\partial W}{\partial r}+\cos \theta \frac{\partial W}{\partial \theta}\right) \\
& +8 W \frac{\sin ^{3} \mu}{g^{2} r^{3}} \sin ^{2} \theta \cos \mu f(1-f)^{2} \\
& +2 \frac{W^{2}}{g^{2} r^{2}} \sin ^{2} \mu(1-f)^{2}\left(\cos ^{2} \mu \sin ^{4} \theta+2 \cos ^{2} \theta\right) \\
& +\frac{1}{2 g^{\prime 2} r^{2}}\left(\sin \theta r \frac{\partial Y}{\partial r}+\cos \theta \frac{\partial Y}{\partial \theta}\right)^{2} \\
& +\frac{\nu^{2}}{2} \frac{\partial h^{2}}{\partial r}+\frac{\nu^{2}}{2 r^{2}} \frac{\partial h^{2}}{\partial \theta}+\frac{\lambda \nu^{4}}{4}\left(1-h^{2}\right)^{2} \\
& +\frac{\nu^{2} h^{2}}{2}\left(\frac{1}{4}(W-Y)^{2}+\frac{2}{r^{2}} \sin ^{2} \mu(1-f)^{2}-\frac{1}{r} \sin \mu \cos \mu(W-Y)(1-f) \sin ^{2} \theta\right)
\end{aligned}
$$

We can rewrite some of these terms as a sum of squares,

$$
\begin{aligned}
& \frac{8}{r^{4} g^{2}} \sin ^{4} \mu(1-f)^{2} f^{2}+8 W \frac{\sin ^{3} \mu}{g^{2} r^{3}} \sin ^{2} \theta \cos \mu f(1-f)^{2} \\
& +2 \frac{W^{2}}{g^{2} r^{2}} \sin ^{2} \mu(1-f)^{2}\left(\cos ^{2} \mu \sin ^{4} \theta+2 \cos ^{2} \theta\right) \\
& =\frac{2}{r^{4} g^{2}} \sin ^{2} \mu(1-f)^{2}\left(\left[2 f \sin \mu+r W \sin ^{2} \theta \cos \mu\right]^{2}+2 r^{2} W^{2} \cos ^{2} \theta\right)
\end{aligned}
$$

Thus in the string core where we have the background fields $h=0, \mathrm{~W} \sim$ constant, the energy density is manifestly a sum of positive definite terms and we can place a lower bound on the energy density,

$$
\mathcal{E}>4 \frac{\partial f^{2}}{\partial r} \frac{\sin ^{2} \mu}{g^{2} r^{2}}+\frac{2}{r^{2} g^{2}} \sin ^{2} \mu(1-f)^{2}\left(2 W^{2} \cos ^{2} \theta\right)
$$


We can place a lower bound on the energy of the configuration by considering the integral of the energy density in regions within $45^{\circ}$ of the poles, i.e. regions with $\cos ^{2} \theta>1 / 2$. A lower bound to this polar contribution to the energy is then obtained by setting $\cos ^{2} \theta=1 / 2$, leading to

$$
E_{\text {polar }}>[2 \pi][2(1-1 / \sqrt{2})] \int r^{2} d r\left(4 \frac{\partial f^{2}}{\partial r} \frac{\sin ^{2} \mu}{g^{2} r^{2}}+\frac{2}{r^{2} g^{2}} \sin ^{2} \mu(1-f)^{2} W^{2}\right)
$$

Recalling that $\mathrm{W}$ is a constant inside the region of symmetry restoration and imposing the boundary conditions $f \rightarrow 0$ as $r \rightarrow 0$ and $f \rightarrow 1$ as $r \rightarrow \infty$, the integral is extremised by taking $f=1-\exp (-|W| r / \sqrt{2})$ and then has the value $2 \sqrt{2} \sin ^{2} \mu|W| / g^{2}$. This gives the bound

$$
E_{\text {polar }}>4 \pi(\sqrt{2}-1) \sin ^{2} \mu \frac{I}{\pi} \frac{1}{G} \log \left(\frac{\gamma}{\beta}\right) \sim E_{\text {Sphal }} \sin ^{2} \mu\left(M_{H} / \mathrm{GeV}\right)\left(I / 10^{10} \operatorname{amps}\right) \log \left(\frac{\gamma}{\beta}\right)
$$

Thus there is a lower limit to the sphaleron energy determined by the string current and the sphaleron energy is increased at large string currents. The origin of this increase is indicated by the above analysis: in the core of the sphaleron the transformation is not pure gauge and so physical gauge fields are created. In the string background these gauge fields acquire a mass through the nonabelian terms in the $\mathrm{SU}(2)$ field strength which couple them to the $\mathrm{Z}$ field. Exciting these fields then entails an energy cost of order the induced mass.

Although this result has been determined for a specific sphaleron configuration, the above discussion suggests that a similar result will hold for any configuration that excites massive gauge fields. With the above form for the background field, $W_{t}^{ \pm}, W_{x}^{ \pm}$and $W_{y}^{ \pm}$ get masses of order I. Working temporarily in cartesian coordinates, the change in baryon number can be rewritten as,

$$
\int d^{4} x W^{a \mu \nu} \tilde{W}_{\mu \nu}^{a}=\int d^{4} x \partial_{\mu}\left(\epsilon^{\mu \nu \lambda \sigma}\left(W_{\nu \lambda}^{a} W_{\sigma}^{a}-\frac{2}{3} g \epsilon^{a b c} W_{\nu}^{a} W_{\lambda}^{b} W_{\sigma}^{c}\right)\right)
$$

If we set the massive gauge fields to zero the second term above vanishes. We can now use the divergence theorem to transform the quantity of interest into a surface integral. The integrand contains a factor of $W_{\nu \lambda}^{a}$ and so vanishes except where the string cuts the surface. The nonvanishing components of the field strength for the string are $W_{13}^{3}$ and $W_{23}^{3}$. Any transformation that produces further components asymptotically will have infinite energy and can be neglected. For a hypercubical surface the faces of interest are $t=t_{i}, t=t_{f}$ and $z \rightarrow-\infty, z \rightarrow+\infty$. The starting and finishing configurations should be identical, so the contributions from the $t=t_{i}, t=t_{f}$ planes should cancel. The contribution from the $z \rightarrow-\infty, z \rightarrow+\infty$ planes is removed by the Levi-Civita tensor. The net change in $\mathrm{B}+\mathrm{L}$ is thus zero.

Alternatively we work directly with the original form. With the massive gauge fields set to zero we have $W_{x y}^{A}=0, W_{t y}^{A}=0$ and $W_{t x}^{A}=0$ where $\mathrm{A}=1,2$ and the quantity of interest reduces to

$$
W^{a \mu \nu} \tilde{W}_{\mu \nu}^{a}=4\left(W_{t x}^{3} W_{y z}^{3}-W_{t y}^{3} W_{x z}^{3}+W_{t z}^{3} W_{x y}^{3}\right)
$$


$=4\left(\left[W_{x, t}^{3}-W_{t, x}^{3}\right]\left[W_{z, y}^{3}-W_{y, z}^{3}\right]-\left[W_{y, t}^{3}-W_{t, y}^{3}\right]\left[W_{z, x}^{3}-W_{x, z}^{3}\right]+\left[W_{z, t}^{3}-W_{t, z}^{3}\right]\left[W_{y, x}^{3}-W_{x, y}^{3}\right]\right)$

The quantity we are interested in is the integral of the above over all space and the time interval of the transition. Integration by parts allows us to exchange the derivatives in each term at the expense of some boundary terms. This takes two integrations by parts and so preserves the sign of each term, however the alternating nature of the Levi-Civita tensor means that the object is odd under interchange of two indices, hence the integrand acquires an overall factor of -1 . Some of the boundary terms contain factors of the form $W_{x y}^{3}$, if the energy of the configuration is finite these must vanish asymptotically. A further integration by parts transforms the remaining terms to this form and they vanish likewise. Thus there are no finite energy baryon number changing paths that do not excite massive gauge fields and we expect the increased barrier height found in the specific example to be a generic feature of the superconducting string background.

We conclude from this argument that the energy barrier separating superconducting string states of different baryon number is large for transitions that occur within the region of electroweak symmetry restoration. This increase in barrier height occurs despite the restoration of the electroweak symmetry and is due to the presence of the large background gauge field. In this case the Higgs sector contributes very little to the energy barrier whilst the pure gauge field contributions are greatly increased.

In the following sections we investigate situations in which the restoration of electroweak symmetry is not a result of a large background gauge field. 


\section{4) The Electroweak String Background}

There are two mechanisms which can cause the restoration of the electroweak symmetry below the critical temperature, the first is via an interaction with some background field, the superconducting string is an example of this, alternatively the electroweak Higgs field may wind and be forced to zero in some region for reasons of continuity, this is the case with electroweak strings. Although the standard model does not admit topologically stable string solutions it has been shown[12] that string solutions do exist and that they are energetically stable for some parameter values[13]. These strings take the form of Nielsen-Olesen vortices[14] formed from the lower component of the Higgs doublet and the $\mathrm{Z}$ field, with all other fields set to zero. The nonvanishing fields are (in cylindrical polar coordinates $(\rho, \phi))$,

$$
\Phi=\left(\begin{array}{c}
0 \\
p \mathrm{e}^{-i \phi}
\end{array}\right) \quad Z_{\phi}=\tilde{Z} / \rho
$$

where for small $\rho: p \propto \rho$ and $\tilde{Z} \propto \rho^{2}$ while at large $\rho: p \rightarrow \nu / \sqrt{2}$ and $\tilde{Z} \rightarrow 2 / G$ $\left(G^{2}=g^{2}+g^{\prime 2}\right)$.

Although electroweak strings are stable for some parameter values, they are unstable for the observed parameter values. This complicates the interpretation of the energies we will calculate for parameter values outside the stability region. In the case of the superconducting string we had a stable background field configuration and we could take the barrier height to be the difference in energy between the pure string configuration and the string plus sphaleron configuration. In the electroweak string case we don't always have a stable background configuration and so in these cases we cannot simply measure the energy difference between the pure string and string plus sphaleron states. For these parameter values we consider the electroweak string background as an example of electroweak symmetry restoration due to the presence of strings from some higher energy phase transition (for example the technicolour model discussed in ref.3). The presence of the higher energy string leads to electroweak symmetry restoration in its vicinity. This pinning of the electroweak Higgs field ameliorates the prime instability of the electroweak string by preventing the upper component of the Higgs field acquiring an expectation value in the core of the string. Whether this is sufficient to stabilise the electroweak string configuration for physical parameter values remains to be investigated. We will assume that the presence of the higher energy string stabilises the electroweak string configuration for parameter values outside the usual stability region. (Alternatively we could imagine the electroweak Higgs field simply pinned to zero in the core of the higher energy string. The scale of symmetry restoration in this case depends on the details of the interaction between the fields. We can't ignore the finite size of the higher energy string as if we simply treat the pinning as a boundary condition at $r=0$, Derricks theorem tells us that the Higgs field expectation value should rise arbitrarily rapidly to its vacuum value.)

Once again we apply the transformation discussed in section 2 , allow the profile functions to depend on $r$ and $\theta$ and evaluate the change in baryon number and the energy density of the static field configurations. From App.A we see that the extra contributions to the change in baryon number from the string gauge field vanish when we integrate over all space: once again we have constructed a baryon number changing path. 
From App.B we see that the energy density is,

$$
\begin{aligned}
\mathcal{E}= & \frac{1}{2 g^{2} r^{2}} \frac{\partial S^{2}}{\partial r}+\frac{1}{2 g^{2} r^{4}}\left(\frac{\partial S}{\partial \theta}+S \cot \theta\right)^{2} \\
& +\frac{1}{2 g^{\prime 2} r^{2}} \frac{\partial Y^{2}}{\partial r}+\frac{1}{2 g^{\prime 2} r^{4}}\left(\frac{\partial Y}{\partial \theta}+Y \cot \theta\right)^{2} \\
& {\left[\frac{\partial h^{2}}{\partial r}+\frac{1}{r^{2}} \frac{\partial h^{2}}{\partial \theta}+\frac{h^{2}}{r^{2}}\left(\frac{S}{2}-\frac{Y}{2}+\frac{1}{\sin \theta}\right)^{2}\right] \frac{\nu^{2}}{2} } \\
& +\frac{\lambda \nu^{4}}{4}\left(1-h^{2}\right)^{2} \\
+\sin ^{2} \mu & 4 \frac{\partial f^{2}}{\partial r} \frac{1}{g^{2} r^{2}}+2 \frac{\partial f^{2}}{\partial \theta} \frac{1}{g^{2} r^{4}}+\frac{2 \sin \theta}{g^{2} r^{2}}\left[\frac{\partial f}{\partial r} \frac{\partial S}{\partial r}+\frac{1}{r^{2}} \frac{\partial f}{\partial \theta} \frac{\partial S}{\partial \theta}\right] \\
& +(6-4 f) \frac{\partial f}{\partial \theta} S \frac{\cos \theta}{g^{2} r^{4}}-4 \frac{\partial S}{\partial \theta}(1-f) f \frac{\cos \theta}{g^{2} r^{4}} \\
& -\frac{4 \cos ^{2} \theta}{g^{2} r^{4} \sin \theta} S f(1-f)+2 S^{2} \frac{\cos ^{2} \theta}{g^{2} r^{4}}(1-f)^{2} \\
& \left.-\frac{h^{2} \nu^{2}}{2 r^{2}}[2 f+\sin \theta(S-Y)](1-f)\right] \\
& \frac{2}{r^{4} g^{2}}[2 f+S \sin \theta]^{2}(1-f)^{2}
\end{aligned}
$$

where $S=g r W_{\phi}^{3}$ and $Y=g^{\prime} r Y_{\phi}$ Rewriting the gauge fields in terms of the photon and Z fields, $G A=g^{\prime} W^{3}+g Y$ and $G Z=g^{\prime} Y-g W^{3}$, asymptotically we have only kinetic terms for $A$. Thus we have the usual forms for the electroweak string gauge fields at large distances from the string core, $A=0$ and $Z_{\phi}=2 /(G r \sin \theta)$. In terms of the original SU(2) and hypercharge fields the asymptotic forms are,

$$
W_{\phi}^{3}=-\frac{g}{G} \frac{2}{G r \sin \theta}, \quad Y_{\phi}=\frac{g^{\prime}}{G} \frac{2}{G r \sin \theta}
$$

or equivalently,

$$
S=-\frac{g^{2}}{G^{2}} \frac{2}{\sin \theta}, \quad Y=\frac{g^{\prime 2}}{G^{2}} \frac{2}{\sin \theta}
$$

The expression for the energy density simplifies if we define

$$
S=\frac{T}{\sin \theta}, \quad Y=\frac{V}{\sin \theta}
$$

Working in cylindrical polar coordinates with the scaled variables $k \rho=r \sin \theta$ and $k z=$ $r \cos \theta$, the energy density then becomes, 


$$
\begin{aligned}
& g^{2} k^{4} \mathcal{E}=\frac{1}{2 \rho^{2}}\left(T,_{\rho}^{2}+T,_{z}^{2}\right)+\frac{g^{2}}{2 g^{\prime 2} \rho^{2}}\left(V,,_{\rho}^{2}+V,_{z}^{2}\right) \\
& {\left[\left(h,_{\rho}^{2}+h,_{z}^{2}\right)+\frac{h^{2}}{\rho^{2}}\left(\frac{T}{2}-\frac{V}{2}+1\right)^{2}\right] \frac{g^{2} k^{2} \nu^{2}}{2}+\frac{\lambda \nu^{4} g^{2} k^{4}}{4}\left(1-h^{2}\right)^{2}} \\
& +\sin ^{2} \mu\left[\frac{2}{r^{2}}\left(f,,_{\rho}^{2}+f,_{z}^{2}\right)+\frac{2}{r^{2}}\left[\cos ^{2} \theta f,_{z}^{2}+2 \cos \theta \sin \theta f,,_{z} f,_{\rho}+\sin ^{2} \theta f,,_{\rho}^{2}\right]+\frac{2}{r^{2}}\left[f,_{\rho} T,,_{\rho}+f,_{z} T,,_{z}\right]\right. \\
& +4(1-f) \frac{\cot \theta}{r^{3}}\left[T(-\sin \theta f, z+\cos \theta f, \rho)-f\left(-\sin \theta T,_{z}+\cos \theta T,_{\rho}\right)\right] \\
& \left.+2 T^{2} \frac{\cos ^{2} \theta}{\sin ^{2} \theta} \frac{1}{r^{4}}(1-f)^{2}-\frac{h^{2} \nu^{2} g^{2} k^{2}}{2 r^{2}}[2 f+T-V](1-f)\right] \\
& +\sin ^{4} \mu \frac{2}{r^{4}}[2 f+T]^{2}(1-f)^{2}
\end{aligned}
$$

Numerical minimisation of this integral gives an energy shift relative to the pure string background of order $50 \%$ of the ordinary sphaleron energy if we set $\sin \theta_{W}$ close to one (in this case the electroweak string is stable). We might expect such a reduction in the energy barrier as the energy contribution from the Higgs sector will be small since the region of electroweak symmetry restoration around the string is of an appropriate size to accommodate the Higgs profile of the sphaleron. If we set $\sin \theta_{W}$ to its observed value, the energy shift relative to the string background at $\sin \mu=\pi / 2$ is of order $10 \%$ of the ordinary sphaleron energy. As we saw in section 2, the Higgs sector contributes less than half of the energy of the sphaleron, thus there is another effect at work in this case. This second effect is the interaction of the string gauge fields with the sphaleron gauge fields. Recalling that $\mathrm{T}$ is negative while $f$ and $\mathrm{V}$ are positive we see that there is the potential for partial cancellation of several terms leading to a further reduction in the sphaleron energy. This observation raises the question of interpretation: one mode of instability in the electroweak string is the formation of a $W$ condensate in the string core[15], is this being excited here? The gauge fields have the appropriate angular dependence, so we are once again faced with the question of where to measure the barrier height from.

We have constructed a baryon number violating transition in the background of an electroweak string. Once again the gauge fields of the string play an important role in determining the energy barrier between states of different baryon number. In this case there may be a considerable decrease in the sphaleron energy, but a detailed analysis of the couplings between the electroweak sector and the higher energy sector producing the strings is required before a definite conclusion can be drawn. 


\section{5) An Alternative Transformation In the Superconducting String Background}

The paths we have considered so far are based on the matrix $U$ discussed in section 2 . This matrix was constructed so as to produce a spherically symmetric sphaleron. When we introduce strings the natural symmetry is cylindrical rather than spherical and we might expect the sphaleron to deform. For example we might consider the sphaleron becoming prolate. As an extreme case we can take the transformation on the equatorial plane of sphaleron and apply this along the whole length of the string i.e. we take $U$ and set $\theta=\pi / 2$, giving the transformation

$$
U^{\prime}=\left(\begin{array}{cc}
\mathrm{e}^{i \mu} \cos \mu & \sin \mu \mathrm{e}^{i \phi} \\
-\sin \mu \mathrm{e}^{-i \phi} & \mathrm{e}^{-i \mu} \cos \mu
\end{array}\right) .
$$

Applying this transformation to the superconducting string background, we find that the change in $\mathrm{B}+\mathrm{L}$ is given by

$$
\begin{aligned}
c \Delta(B+L)=\int d^{4} x \quad 2 \dot{\mu} \frac{\sin \mu \cos \mu}{g^{2} r^{3} \sin \theta}( & \frac{\partial}{\partial \theta}\left(S \cos \theta(f-1)^{2}\right)+(f-1) \frac{\partial}{\partial \theta}(S \cos \theta) \\
& \left.+r \sin \theta\left[\frac{\partial}{\partial r}\left(S(f-1)^{2}\right)+(f-1) \frac{\partial}{\partial r} S\right]\right)
\end{aligned}
$$

where $S=g r W_{z}^{3}$ and $c=-2 \pi^{2} / N_{f} g^{2}$. The string gauge field vanishes at the centre of the string, so the first term vanishes when integrated with respect to $\theta$. After integrating the second term by parts with respect to $\theta$ and the remaining terms with respect to $r$ we have

$$
\begin{aligned}
c \Delta(B+L)=4 \pi \int d t \dot{\mu} \frac{\sin \mu \cos \mu}{g^{2}}( & \int d r d \theta(-S \cos \theta) \frac{1}{r} \frac{\partial f}{\partial \theta} \\
& +\int d \theta \sin \theta\left[S(f-1)^{2}+(f-1) S\right]_{0}^{\infty} \\
& \left.-\int d r d \theta \sin \theta S \frac{\partial f}{\partial r}\right)
\end{aligned}
$$

Now, $\cos \theta \frac{1}{r} \frac{\partial f}{\partial \theta}+\sin \theta \frac{\partial f}{\partial r}=\frac{\partial f}{\partial \rho}$ where $\rho$ is the radial coordinate in cylindrical polars.

$$
c \Delta(B+L)=4 \pi \int d t \dot{\mu} \frac{\sin \mu \cos \mu}{g^{2}}\left(\int d r d \theta\left(-S \frac{\partial f}{\partial \rho}\right)+\int d \theta \sin \theta\left[S(f-1)^{2}+(f-1) S\right]_{0}^{\infty}\right)
$$

As $\mathrm{S}$ vanishes at $r=0$, the lower limit of the boundary term gives zero. Far from the string $f$ tends to one, so the only contribution from the upper limit comes from the string core. $\left[S(f-1)^{2}+(f-1) S\right]$ is finite and only nonzero in regions with $\sin \theta<\rho_{c} / R$ where $\rho_{c}$ is the core radius and $R$ is the spherical distance we are considering. As $R \rightarrow \infty$ the contribution from the string core vanishes and we are left with

$$
c \Delta(B+L)=4 \pi \int d t \dot{\mu} \frac{\sin \mu \cos \mu}{g^{2}} \int d r d \theta\left(-S \frac{\partial f}{\partial \rho}\right)
$$


$\mathrm{S}$ has a fixed sign and $f$ is monotonically increasing away from the string core, thus the integrand has a fixed sign and the spatial integrals are nonzero. The temporal integral gives a factor of $[-\cos (2 \mu) / 2]_{i}^{f}$, thus the vacuum $\left(\mu_{i}=0\right)$ to vacuum $\left(\mu_{f}=\pi\right)$ transition gives no net change in $\mathrm{B}+\mathrm{L}$. However, there is a change in $\mathrm{B}+\mathrm{L}$ as we move from the vacuum $\left(\mu_{i}=0\right)$ to $\mu=\pi / 2$. The field configurations at $\mu=\pi / 2$ are

$$
\begin{gathered}
\tilde{\Phi}=\frac{v}{\sqrt{2}}\left(\begin{array}{c}
\mathrm{e}^{i \phi} \\
0
\end{array}\right) p(\rho) \\
W_{\mu}=U^{\prime} \delta_{\mu, z} \tau^{3} \frac{S}{g r} U^{\prime-1}-\frac{f}{g} \partial_{\mu} U^{\prime} U^{\prime-1}=-\delta_{\mu, z} \tau^{3} \frac{S}{g r}-\frac{f}{g} \delta_{\phi \mu}\left(\begin{array}{cc}
i & 0 \\
0 & -i
\end{array}\right)
\end{gathered}
$$

Thus we have the superconducting string superimposed on an electroweak Higgs field with a winding number, nonvanishing $W_{z}^{3}$ and $W_{\theta}^{3}$ but vanishing $W_{\mu}^{ \pm}$.

The energy density for static configurations generated by this transformation is

$$
\begin{aligned}
\mathcal{E}= & \frac{1}{2 \sin ^{2} \theta} \frac{1}{r^{4} g^{2}}\left(4 \sin ^{2} \mu\left(\frac{\partial f}{\partial \theta}^{2}+r^{2} \frac{\partial f}{\partial r}^{2}\right)+r^{2} \sin ^{4} \theta \frac{\partial S^{2}}{\partial r}\right. \\
& -2 \frac{\partial S}{\partial r} r S \sin ^{4} \theta+\frac{\partial S}{\partial \theta}^{2} \cos ^{2} \theta \sin ^{2} \theta+2 \cos \theta \sin ^{3} \theta \frac{\partial S}{\partial \theta}\left(r \frac{\partial S}{\partial r}-S\right) \\
& \left.+S^{2}\left[\sin ^{4} \theta+4 \cos ^{2} \mu(1-f)^{2} \sin ^{2} \mu\right]\right) \\
& +\frac{\nu^{2}}{2}\left[\frac{\partial h^{2}}{\partial r}+\frac{1}{r^{2}} \frac{\partial h^{2}}{\partial \theta}+\frac{h^{2}}{r^{2}} \frac{1}{\sin ^{2} \theta}(1-f)^{2} \sin ^{2} \mu+\frac{h^{2}}{4 r^{2}}(S-Y)^{2}\right] \\
& +\frac{\lambda \nu^{4}}{4}\left(1-h^{2}\right)^{2}+\frac{1}{2 r^{4} g^{\prime 2}}\left(\frac{\partial Y}{\partial \theta} \cos \theta+\left(\frac{\partial Y}{\partial r}-Y / r\right) \sin \theta r\right)^{2}
\end{aligned}
$$

where $Y=g^{\prime} r Y_{z}$. The interesting feature in this case is the form of the $S^{2}$ term. The contribution due to the transformation has the form $S^{2}(1-f)^{2} \cos ^{2} \mu \sin ^{2} \mu$, thus it vanishes at $\mu=\pi / 2$. This is what we would expect from our discussion in section 3 given the absence of $W^{ \pm}$fields in this case. We thus expect to incur a large energy cost if we try to deform away from this configuration. Although a detailed stability analysis would be required to prove the stability of the electroweak sector with this winding, this observation does suggest that the winding might be stable. The background gauge field generated by the current reduces the two main modes of instability of the electroweak string. Given that electroweak strings are themselves superconducting, it would be interesting if the electroweak string could carry its own stabilising current.

In the background of a superconducting string it is possible that windings in the electroweak Higgs field are stabilised and such configurations carry a net baryon plus lepton number. 


\section{Conclusions}

We have constructed baryon number changing transitions in various string backgrounds. The energy barriers in the various backgrounds depend crucially on the region of symmetry restoration and the background gauge fields. For a simple dip in the vacuum expectation value of the electroweak Higgs field, generated for instance by a scalar coupling to a higher energy string, the sphaleron barrier can be reduced to about half its standard size although the precise reduction in the barrier height depends on the details of the model. In the background of an electroweak string the gauge fields can be exploited to further lower the sphaleron barrier, unfortunately the interpretation of this result is complicated by considerations of the stability of the string. However, in the case of the superconducting cosmic string background the gauge fields that produce the large region of electroweak symmetry restoration also generate masses for the $W^{ \pm}$fields, increasing the height of the sphaleron energy barrier.

It is possible to reduce the sphaleron energy barrier in the background of a cosmic string, but the scale of the reduction depends on the details of the model and background gauge fields can even increase the barrier height. This causes problems for the baryogenesis mechanism of ref.3 that is based on superconducting cosmic strings. The mechanism based on technistrings may be viable, but a detailed model of the electroweak background fields is required.

In the presence of a superconducting cosmic string windings of the electroweak Higgs field may be stabilised and such configurations carry baryon number.

\section{Acknowledgements}

I would like to acknowledge A.C.Davis for interesting me in this project and N.Dorey and S.Hands for useful discussions.

\section{References}

[1] N.Turok and T.Zadrozny Phys.Rev.Lett65 (1990) 2331

L.McLerran, M.Shaposhnikov, N.Turok and M.Voloshin Phys.Lett.B256 (1991) 451

A.Cohen, D.Kaplan and A.Nelson Phys.Lett.B263 (1991) 86

A.Nelson, D.Kaplan and A.Cohen Nucl.Phys.B373 (1992) 453

[2] M.Dine, R.Leigh, P.Huet, A.Linde and D.Linde Phys.Rev.D46 (1992) 550

[3] R.Brandenberger, A.C.Davis and M.Trodden Phys.Lett.335 (1994) 123

[4] P.Arnold and L.McLerran Phys.Rev.D36 (1987) 581, Phys. Rev. D37 (1988) 1020

[5] F.R.Klinkhamer and N.Manton Phys.Rev.D30 (1984) 2212

[6] M.James Phd Thesis DAMTP Cambridge (1993)

[7] J.Ambjørn, M.Laursen and M.Shaposhnikov Phys.Lett.B197 (1987) 49

J.Ambjørn, T.Askgaard, H.Porter and M.Shaposhnikov Nucl.Phys.B353 (1991) 346

[8] P.Damgaard and D.Espriu Phys.Lett.B256 (1991) 442

[9] W.B.Perkins and A.C.Davis Nucl.Phys.B406 (1993) 377

[10] M.Trodden Mod. Phys.Lett.A9 (1994) 2649

[11] W.B.Perkins Nucl.Phys.B364 (1991) 451 
[12] T.Vachaspati Phys.Rev.Lett.68 (1992) 1977

Y.Nambu Nucl.Phys.B130 (1977) 505

[13] M.James, L.Perivolaropoulos and T.Vachaspati Nucl.Phys.B395 (1993) 534

[14] H.B.Nielsen and P.Olesen Nucl.Phys.B61 (1973) 45

[15] W.B.Perkins Phys.Rev.D47 (1993) 5224 


\section{Appendix A. Calculating the Change in $\mathrm{B}+\mathrm{L}$}

\section{The Ordinary Sphaleron}

The quantity that we need to calculate is $\int d^{4} x W \tilde{W}$ where $\tilde{W}$ is the dual of $\mathrm{W}$, $\tilde{W}^{\mu \nu}=\frac{1}{2} \epsilon^{\mu \nu \sigma \rho} W_{\sigma \rho}$. Using the symmetry of the alternating tensor and of the field strength, we have three distinct contributions to calculate:

$$
W_{t r} W_{\theta \phi} \quad, \quad W_{t \theta} W_{r \phi} \quad \text { and } \quad W_{t \phi} W_{r \theta}
$$

Using the pure sphaleron gauge fields

$$
W_{\mu}=-\frac{f(r)}{g}\left(\partial_{\mu} U\right) U^{-1}
$$

with the aid of Maple we find

$$
\operatorname{Tr}\left(W_{t r} W_{\theta \phi}-W_{t \theta} W_{r \phi}+W_{t \phi} W_{r \theta}\right)=12 \frac{\partial f}{\partial r} f \sin ^{2}(\mu)(1-\cos (\theta)) \frac{f-1}{g^{2} r^{2}}
$$

Integrating over space we have

$$
\begin{gathered}
\int_{0}^{2 \pi} d \phi \int_{0}^{\pi} \sin \theta d \theta \int_{0}^{\infty} r^{2} d r \operatorname{Tr}\left(W_{t r} W_{\theta \phi}-W_{t \theta} W_{r \phi}+W_{t \phi} W_{r \theta}\right) \\
=\frac{12 \sin ^{2}(\mu)}{g^{2}} \int_{0}^{2 \pi} d \phi \int_{0}^{\pi} \sin \theta d \theta(1-\cos (\theta)) \int_{0}^{\infty} d r \frac{\partial f}{\partial r} f(f-1) \\
=\frac{12 \sin ^{2}(\mu)}{g^{2}}[2 \pi][2]\left[\frac{f^{3}}{3}-\frac{f^{2}}{2}\right]_{0}^{\infty}=-\frac{8 \pi}{g^{2}} \sin ^{2} \mu
\end{gathered}
$$

where we have used the boundary conditions on $f, f(0)=0$ and $f(\infty)=1$. Thus we have

$$
\begin{gathered}
\Delta(B+L)=\frac{N_{f}}{16 \pi^{2}} \int d^{4} x g^{2} W_{a}^{\mu \nu} \tilde{W}_{a \mu \nu}=\frac{N_{f}}{16 \pi^{2}} \int d^{4} x g^{2} \frac{1}{2} \epsilon^{\mu \nu \lambda \rho} W_{a \mu \nu} W_{a \lambda \rho} \\
=\frac{4 N_{f}}{\pi} \int_{0}^{\pi} d \mu \sin ^{2}(\mu)=2 N_{f}
\end{gathered}
$$

where we have used the normalisation condition, $\operatorname{Tr}\left(t^{a} t^{b}\right)=-\frac{1}{2} \delta^{a b}$.

Now that we have calculated the pure sphaleron contribution to the change in $B+L$ we can see what changes the string backgrounds make.

\section{The Superconducting String Background}

In the case of superconducting strings we have the gauge field discussed in section 3 ,

$$
\vec{W}_{\mu}=U \delta_{\mu, z} \tau^{3} \frac{W(r, \theta)}{g} U^{-1}-\frac{f(r, \theta)}{g}\left(\partial_{\mu} U\right) U^{-1}
$$


where $W$ is a constant inside the region of symmetry restoration. Substituting these fields into $\operatorname{Tr}\left(W_{t r} W_{\theta \phi}-W_{t \theta} W_{r \phi}+W_{t \phi} W_{r \theta}\right)$ we find

$$
\begin{aligned}
\operatorname{Tr}\left(W_{t r} W_{\theta \phi}-W_{t \theta} W_{r \phi}+W_{t \phi} W_{r \theta}\right)= & 12(\cos (\theta)-1) \sin ^{2}(\mu) \frac{\partial f}{\partial r} \frac{f(1-f)}{r^{2} g^{2}} \\
& +\sin (\mu) \cos (\mu)(\mu \quad \text { indep. })
\end{aligned}
$$

The term that is independent of $\mu$ carries no explicit $\mu$ dependence, but does contain various profile functions. We can calculate the change in baryon number with fixed profiles satisfying the appropriate boundary conditions. In this case the profile functions do not provide any implicit $\mu$ dependence and the extra term does not contribute to the overall change in baryon number. At any stage during the transition the profile functions will differ from those that give the lowest energy density, but we can make local deformations of the fields to obtain the optimal profiles. These deformations do not change the asymptotic values of the fields and so do not alter $\Delta_{B+L}$.

The first term,

$$
12(\cos (\theta)-1) \sin ^{2}(\mu) \frac{\partial f}{\partial r} \frac{f(1-f)}{r^{2} g^{2}}
$$

is simply the contribution from the ordinary sphaleron, thus the string background fields do not alter $\Delta_{B+L}$.

\section{The electroweak string background}

In this case we apply our transformation to a background field consisting of a straight electroweak string oriented along the polar axis. The only $\mathrm{W}$ field excited in the string background is $W_{\phi}^{3}$. We denote this field by

$$
W_{\phi}^{3}=\frac{S(r, \theta)}{g r}
$$

where $S \rightarrow 0$ close to the string core and $S \rightarrow$ constant far from the core. The gauge field during the transition is then

$$
\vec{W}_{\mu}=U \delta_{\mu, \phi} \tau^{3} \frac{S(r, \theta)}{g r} U^{-1}-\frac{f(r, \theta)}{g}\left(\partial_{\mu} U\right) U^{-1}
$$

Evaluating $W \tilde{W}$ we find,

$$
\begin{aligned}
\operatorname{Tr}\left(W_{t r} W_{\theta \phi}\right. & \left.-W_{t \theta} W_{r \phi}+W_{t \phi} W_{r \theta}\right)=\operatorname{Tr}\left(W_{t r} W_{\theta \phi}-W_{t \theta} W_{r \phi}+W_{t \phi} W_{r \theta}\right)_{\text {ord.sph }} \\
& +\frac{\sin (\theta)}{g^{2} r^{2}(1+\cos (\theta))}(1-f)\left(-4 \frac{\partial f}{\partial r} S-2 f \frac{\partial S}{\partial r}\right) \sin ^{2}(\theta) \sin ^{2}(\mu) \\
& +\frac{\sin \theta}{g^{2} r^{2}(1+\cos (\theta))} \frac{\partial f}{\partial r} S\left(\cos ^{2}(\theta) \sin ^{2}(\mu)-\cos (\theta) \cos ^{2}(\mu)\right) \\
& +\frac{1}{g^{2} r^{2}}\left(\frac{\partial f}{\partial \theta} \frac{\partial S}{\partial r}-\frac{\partial f}{\partial r} \frac{\partial S}{\partial \theta}\right)\left(1-\cos (\theta)-\sin ^{2}(\theta) \sin ^{2}(\mu)\right)
\end{aligned}
$$


Integrating over $\mu$ for fixed profile functions we find that the extra contribution from the string background fields is,

$$
\begin{aligned}
\frac{1}{\pi} \int d t T r\left(W_{t r} W_{\theta \phi}-\right. & \left.W_{t \theta} W_{r \phi}+W_{t \phi} W_{r \theta}\right)_{E W}= \\
& \frac{\sin (\theta)}{g^{2} r^{2}(1+\cos (\theta))}(1-f)\left(-2 \frac{\partial f}{\partial r} S-f \frac{\partial S}{\partial r}\right) \sin ^{2}(\theta) \\
& +\frac{\sin \theta}{2 g^{2} r^{2}(1+\cos (\theta))} \frac{\partial f}{\partial r} S\left(\cos ^{2}(\theta)-\cos (\theta)\right) \\
& +\frac{1}{g^{2} r^{2}}\left(\frac{\partial f}{\partial \theta} \frac{\partial S}{\partial r}-\frac{\partial f}{\partial r} \frac{\partial S}{\partial \theta}\right)\left(1-\cos (\theta)-\frac{1}{2} \sin ^{2}(\theta)\right)
\end{aligned}
$$

Now, $\frac{\partial f}{\partial \theta} \frac{\partial S}{\partial r}-\frac{\partial f}{\partial r} \frac{\partial S}{\partial \theta}=\left(S \frac{\partial f}{\partial \theta}\right)_{, r}-\left(S \frac{\partial f}{\partial r}\right)_{, \theta}$ and integrating the first term with respect to $r$ gives $\left.S \frac{\partial f}{\partial \theta}\right|_{0} ^{\infty}$ which vanishes due to the boundary conditions on $f$. Similarly, if we integrate the second term by parts with respect to $\theta$ the boundary term vanishes as $\theta=0$ and $\theta=\pi$ both correspond to points in the string core and $S=0$ at the centre of the string. Thus we are left with

$$
\begin{aligned}
& \frac{1}{\pi} \int d t \int r^{2} d r \int \sin (\theta) d \theta \operatorname{Tr}\left(W_{t r} W_{\theta \phi}-W_{t \theta} W_{r \phi}+W_{t \phi} W_{r \theta}\right)_{E W}= \\
& \int d r d \theta\left(\frac{\sin ^{2} \theta}{g^{2}(1+\cos \theta)}(1-f)\left(-2 \frac{\partial f}{\partial r} S-f \frac{\partial S}{\partial r}\right) \sin ^{2} \theta\right. \\
& \quad+\frac{\sin ^{2} \theta}{2 g^{2}(1+\cos (\theta))} \frac{\partial f}{\partial r} S\left(\cos ^{2}(\theta)-\cos (\theta)\right) \\
& \left.\quad+\frac{1}{g^{2}} S \frac{\partial f}{\partial r} \partial_{\theta}\left[\sin \theta\left(1-\cos (\theta)-\frac{1}{2} \sin ^{2}(\theta)\right)\right]\right)
\end{aligned}
$$

Further, using the identity

$$
\frac{\partial}{\partial r}\left((1-f)^{2} S\right)=(1-f)\left(-2 \frac{\partial f}{\partial r} S+(1-f) \frac{\partial S}{\partial r}\right)=(1-f)\left(-2 \frac{\partial f}{\partial r} S-f \frac{\partial S}{\partial r}\right)+(1-f) \frac{\partial S}{\partial r}
$$

we can integrate the first term by parts with respect to $r$,

$\int d r(1-f)\left(-2 \frac{\partial f}{\partial r} S-f \frac{\partial S}{\partial r}\right)=\left((1-f)^{2} S\right)_{0}^{\infty}-\left[(1-f) S-\int d r\left(-\frac{\partial f}{\partial r}\right) S\right]_{0}^{\infty}=\int d r\left(-\frac{\partial f}{\partial r}\right) S$ where we have made use of the boundary conditions on $f$ and $S$. This then gives

$$
\begin{aligned}
& \frac{1}{\pi} \int d t \int r^{2} d r \int \sin (\theta) d \theta \operatorname{Tr}\left(W_{t r} W_{\theta \phi}-W_{t \theta} W_{r \phi}+W_{t \phi} W_{r \theta}\right)_{E W}=\frac{1}{g^{2}} \int d r d \theta \frac{\partial f}{\partial r} S \times \\
&\left(-(1-\cos \theta) \sin ^{2} \theta+\frac{1}{2}(1-\cos \theta)\left(\cos ^{2} \theta-\cos \theta\right)\right. \\
&\left.+\cos \theta\left(1-\cos \theta-\frac{1}{2} \sin ^{2} \theta\right)+\sin \theta(\sin \theta-\sin \theta \cos \theta)\right) \\
&=\frac{1}{g^{2}} \int d r d \theta \frac{\partial f}{\partial r} S(1-\cos \theta)\left(-\sin ^{2} \theta-\frac{1}{2}(1-\cos \theta) \cos \theta+\cos \theta\left(1-\frac{1}{2}(1+\cos \theta)\right)+\sin ^{2} \theta\right)=0
\end{aligned}
$$

Thus there is no contribution to the net change in baryon number from the gauge field of the string. 


\section{Appendix 2: The Sphaleron Energy}

\section{The Ordinary Sphaleron}

We can find the form of the energy barrier separating the two vacua that are the end points of our path by considering static field configurations on this path. That is we look at the energy of configurations at constant $\mu$. In this case the gauge fields have no temporal components and all time derivatives vanish. We can use the components of the field strength given in appendix A to evaluate the gauge field contribution to the sphaleron energy:

$$
\begin{aligned}
\Theta_{W W}^{00} \equiv \frac{1}{4} W_{\mu \nu}^{a} W^{a \mu \nu} & =-\frac{1}{2} \operatorname{Tr} W_{\mu \nu} W^{\mu \nu}=-\operatorname{Tr}\left(W_{r \theta} W^{r \theta}+W_{r \phi} W^{r \phi}+W_{\theta \phi} W^{\theta \phi}\right) \\
& =4\left(\frac{\partial f}{\partial r}\right)^{2} \frac{\sin ^{2} \mu}{g^{2} r^{2}}+\frac{8}{r^{4} g^{2}} \sin ^{4} \mu(1-f)^{2} f^{2}
\end{aligned}
$$

The Higgs field covariant derivative terms are found using the explicit forms for the gauge fields and the Higgs field discussed in section 2.

$$
\begin{gathered}
\left(D_{\mu} \Phi\right)^{\dagger} D^{\mu} \Phi=\sin ^{2} \mu\left(\frac{\partial h^{2}}{\partial r}+\frac{1}{r^{2}}\left[2 f \cos ^{2} \mu(\right.\right. \\
\left.\left(1-h^{2}\right)-2 h(1-h)\right) \\
\left.\left.+2 h^{2}(1-f)^{2}\right]\right) \frac{\nu^{2}}{2}
\end{gathered}
$$

Finally the Higgs potential term is given by

$$
\lambda\left(\phi^{2}-\nu^{2} / 2\right)^{2}=\lambda \frac{\nu^{4}}{4} \sin ^{4} \mu\left(1-h^{2}\right)^{2}
$$

Thus the energy density of the sphaleron $(\mu=\pi / 2)$ is given by

$$
\begin{aligned}
\mathcal{E}_{\text {sphaleron }}= & \frac{\nu^{2}}{2} \frac{\partial h^{2}}{\partial r}+\frac{\nu^{2}}{r^{2}} h^{2}(1-f)^{2} \\
& +4\left(\frac{\partial f}{\partial r}\right)^{2} \frac{1}{g^{2} r^{2}}+\frac{8}{r^{4} g^{2}}(1-f)^{2} f^{2}+\lambda \frac{\nu^{4}}{4}\left(1-h^{2}\right)^{2}
\end{aligned}
$$

The sphaleron energy is thus given by[5]

$$
\begin{gathered}
E_{\text {sphaleron }}=\int d^{3} x \mathcal{E}_{\text {sphaleron }} \\
=[2 \pi][2] \int d r\left[\frac{\nu^{2}}{2} r^{2} \frac{\partial h^{2}}{\partial r}+\nu^{2} h^{2}(1-f)^{2}\right. \\
\left.+4\left(\frac{\partial f}{\partial r}\right)^{2} \frac{1}{g^{2}}+\frac{8}{r^{2} g^{2}}(1-f)^{2} f^{2}+\lambda \frac{\nu^{4}}{4} r^{2}\left(1-h^{2}\right)^{2}\right]
\end{gathered}
$$


If we let $r=\eta x, \partial_{r}=\partial_{x} / \eta$

$$
\begin{aligned}
E_{\text {sphaleron }}=[4 \pi] \eta \int d x & {\left[\frac{\nu^{2}}{2}\left(x^{2} \frac{\partial h^{2}}{\partial x}+2 h^{2}(1-f)^{2}\right)\right.} \\
& +4\left(\frac{\partial f}{\partial x}\right)^{2} \frac{1}{\eta^{2} g^{2}}+\frac{8}{\eta^{2} x^{2} g^{2}}(1-f)^{2} f^{2} \\
& \left.+\lambda \frac{\nu^{4}}{4} \eta^{2} x^{2}\left(1-h^{2}\right)^{2}\right]
\end{aligned}
$$

If we set $\nu^{2}=\kappa^{2} / \eta^{2} g^{2}$ (i.e. $\eta=\kappa / \nu g$ ) we have

$$
\begin{aligned}
E_{\text {sphaleron }}= & 4 \pi \frac{\kappa}{\nu g} \nu^{2} \int d x \\
& {\left[\frac{1}{2} x^{2} \frac{\partial h^{2}}{\partial x}+h^{2}(1-f)^{2}\right.} \\
& \left.+\frac{4}{\kappa^{2}}\left(\frac{\partial f}{\partial x}\right)^{2}+\frac{8}{x^{2} \kappa^{2}}(1-f)^{2} f^{2}+\frac{\lambda}{g^{2}} \frac{\kappa^{2}}{4} x^{2}\left(1-h^{2}\right)^{2}\right]
\end{aligned}
$$

Thus $E_{\text {sphaleron }}=\frac{4 \pi \nu}{g} D\left(\frac{\lambda}{g^{2}}\right)=\frac{8 \pi M_{W}}{g^{2}} D\left(\frac{\lambda}{g^{2}}\right)$ where D is $\kappa$ times the dimensionless integral above. Values for D were calculated by Klinkhamer and Manton[5]. The dimensionless factor $\kappa$ is arbitrary and allows us to estimate the relative contributions of the various terms.

\section{The Superconducting String Case}

In the case of the superconducting string the $\mathrm{SU}(2)$ gauge field strength contribution to the energy density can be found from the explicit forms of the gauge field discussed in appendix A. For static field configurations we have,

$$
\begin{aligned}
\Theta_{W W}^{00}= & 4 \frac{\partial f^{2}}{\partial r} \frac{\sin ^{2} \mu}{g^{2} r^{2}}+\frac{8}{r^{4} g^{2}} \sin ^{4} \mu(1-f)^{2} f^{2}+\frac{2}{g^{2} r^{4}} \sin \mu^{2} \frac{\partial f}{\partial \theta} \\
& +\frac{1}{2 g^{2} r^{2}}\left(\sin \theta\left(r \frac{\partial W}{\partial r}\right)+\cos \theta \frac{\partial W}{\partial \theta}\right)^{2} \\
& +\frac{2}{g^{2} r^{2}} \frac{\partial f}{\partial r} \sin \mu \cos \mu \sin \theta\left(\sin \theta\left(r \frac{\partial W}{\partial r}\right)+\cos \theta \frac{\partial W}{\partial \theta}\right) \\
& +8 W \frac{\sin ^{3} \mu}{g^{2} r^{3}} \sin ^{2} \theta \cos \mu f(1-f)^{2} \\
& +2 \frac{W^{2}}{g^{2} r^{2}} \sin ^{2} \mu(1-f)^{2}\left(\cos ^{2} \mu \sin ^{4} \theta+2 \cos ^{2} \theta\right)
\end{aligned}
$$

If we assume that the hypercharge field takes the form

$$
Y_{\mu}=\delta_{\mu, z} \frac{Y(r, \theta)}{g^{\prime} r},
$$


the contribution from the hypercharge field strength to the energy density is found to be,

$$
\Theta_{Y Y}^{00}=\frac{1}{2 g^{\prime 2} r^{2}}\left(\sin \theta\left(r \frac{\partial Y}{\partial r}\right)+\cos \theta \frac{\partial Y}{\partial \theta}\right)^{2}
$$

The Higgs field in this case has the form

$$
\Phi \rightarrow U \Phi=\frac{\nu}{\sqrt{2}}\left(\begin{array}{c}
\sin \mu \sin \theta \mathrm{e}^{i \phi} \\
\mathrm{e}^{-i \mu}(\cos \mu+i \sin \mu \cos \theta)
\end{array}\right) p(r, \theta)
$$

This form for $\Phi$ gives the following contributions to the energy density, from the covariant derivative term

$$
\begin{aligned}
\left(D_{\mu} \Phi\right)^{\dagger} D^{\mu} \Phi= & {\left[\frac{\partial h^{2}}{\partial r}+\frac{1}{r^{2}} \frac{\partial h^{2}}{\partial \theta}+\frac{h^{2}}{r^{2}}\left(\frac{1}{4}(S-Y)^{2} r^{2}\right.\right.} \\
& \left.\left.+2 \sin ^{2} \mu(1-f)^{2}-\sin \mu \cos \mu r(S-Y)(1-f) \sin ^{2} \theta\right)\right] \frac{\nu^{2}}{2}
\end{aligned}
$$

and from the potential term:

$$
\lambda\left(\frac{\nu^{2}}{2}-\phi^{\dagger} \phi\right)^{2}=\frac{\lambda \nu^{4}}{4}\left(1-h^{2}\right)^{2}
$$

The full energy density is thus,

$$
\begin{aligned}
\mathcal{E}= & \\
& 4 \frac{\partial f^{2}}{\partial r} \frac{\sin ^{2} \mu}{g^{2} r^{2}}+\frac{8}{r^{4} g^{2}} \sin ^{4} \mu(1-f)^{2} f^{2}+\frac{2}{g^{2} r^{4}} \sin \mu^{2} \frac{\partial f^{2}}{\partial \theta} \\
& +\frac{1}{2 g^{2} r^{2}}\left(\sin \theta\left(\frac{\partial W}{\partial r}\right)+\cos \theta \frac{\partial W}{\partial \theta}\right)^{2} \\
& +\frac{2}{g^{2} r^{2}} \frac{\partial f}{\partial r} \sin \mu \cos \mu \sin \theta\left(\sin \theta\left(r \frac{\partial W}{\partial r}\right)+\cos \theta \frac{\partial W}{\partial \theta}\right) \\
& +8 W \frac{\sin ^{3} \mu}{g^{2} r^{3}} \sin ^{2} \theta \cos \mu f(1-f)^{2} \\
& +2 \frac{W^{2}}{g^{2} r^{2}} \sin ^{2} \mu(1-f)^{2}\left(\cos ^{2} \mu \sin ^{4} \theta+2 \cos ^{2} \theta\right) \\
& +\frac{1}{2 g^{\prime 2} r^{2}}\left(\sin \theta\left(r \frac{\partial Y}{\partial r}\right)+\cos \theta \frac{\partial Y}{\partial \theta}\right)^{2} \\
& +\frac{\nu^{2}}{2} \frac{\partial h^{2}}{\partial r}+\frac{\nu^{2}}{2 r^{2}} \frac{\partial h^{2}}{\partial \theta}+\frac{\lambda \nu^{4}}{4}\left(1-h^{2}\right)^{2} \\
& +\frac{\nu^{2} h^{2}}{2 r^{2}}\left(\frac{1}{4}(S-Y)^{2} r^{2}+2 \sin ^{2} \mu(1-f)^{2}-\sin \mu \cos \mu r(S-Y)(1-f) \sin ^{2} \theta\right)
\end{aligned}
$$




\section{Electroweak string case}

The gauge field in this case is discussed in appendix A, the field strength gives:

$$
\begin{aligned}
& \Theta_{W W}^{00}= \frac{1}{2 g^{2} r^{2}} \frac{\partial S^{2}}{\partial r}+\frac{1}{2 g^{2} r^{4}}\left(\frac{\partial S}{\partial \theta}+S \cot \theta\right)^{2} \\
&+\sin ^{2} \mu\left[4 \frac{\partial f}{\partial r} \frac{1}{g^{2} r^{2}}+2 \frac{\partial f}{\partial \theta} \frac{1}{g^{2} r^{4}}+\frac{2 \sin \theta}{g^{2} r^{2}}\left[\frac{\partial f}{\partial r} \frac{\partial S}{\partial r}+\frac{1}{r^{2}} \frac{\partial f}{\partial \theta} \frac{\partial S}{\partial \theta}\right]\right. \\
&+(6-4 f) \frac{\partial f}{\partial \theta} S \frac{\cos \theta}{g^{2} r^{4}}-4 \frac{\partial S}{\partial \theta}(1-f) f \frac{\cos \theta}{g^{2} r^{4}} \\
&\left.-\frac{4 \cos ^{2} \theta}{g^{2} r^{4} \sin \theta} S f(1-f)+2 S^{2} \frac{\cos ^{2} \theta}{g^{2} r^{4}}(1-f)^{2}\right] \\
&+\sin ^{4} \mu \frac{2}{r^{4} g^{2}}[2 f+S \sin \theta]^{2}(1-f)^{2}
\end{aligned}
$$

The hypercharge field strength gives a contribution to the energy density of

$$
\Theta_{Y Y}^{00}=\frac{1}{2 g^{\prime 2} r^{2}} \frac{\partial Y^{2}}{\partial r}+\frac{1}{2 g^{\prime 2} r^{4}}\left(\frac{\partial Y}{\partial \theta}+Y \cot \theta\right)^{2}
$$

The Higgs field takes the from

$$
\Phi \rightarrow U \Phi=\frac{\nu}{\sqrt{2}}\left(\begin{array}{c}
\sin \mu \sin \theta \\
\mathrm{e}^{-i(\mu+\phi)}(\cos \mu+i \sin \mu \cos \theta)
\end{array}\right) p(r, \theta)
$$

which leads to the following contributions to the energy density: from the scalar covariant derivative term:

$$
\begin{aligned}
\left(D_{i} \Phi\right)^{\dagger} D_{i} \Phi= & \left(\frac{\partial h^{2}}{\partial r}+\frac{1}{r^{2}} \frac{\partial h^{2}}{\partial \theta}+\frac{h^{2}}{r^{2}}\left(\frac{S}{2}-\frac{Y}{2}+\frac{1}{\sin \theta}\right)^{2}\right. \\
& \left.-\sin ^{2} \mu \frac{h^{2}}{r^{2}}[2 f+\sin \theta(S-Y)](1-f)\right) \frac{\nu^{2}}{2}
\end{aligned}
$$

and from the scalar potential term:

$$
\lambda\left(\frac{\nu^{2}}{2}-|\Phi|^{2}\right)^{2}=\frac{\lambda \nu^{4}}{4}\left(1-h^{2}\right)^{2}
$$

